\title{
Photodissociation Dynamics of Ortho and Meta Fluorophenols: The Origin of the Fast Protons
}

\author{
Xinli Song, ${ }^{\mathbf{*}}{ }^{\text {Josene M. Toldo }},{ }^{\mathbf{2}^{*}}$ Carlos E. V. de Moura, ${ }^{2}$ Ying Tang, ${ }^{\mathbf{1}}$ Bing Zhang, ${ }^{\mathbf{1}}$ Mario \\ Barbatti $^{2 *}$ \\ 1 State Key Laboratory of Magnetic Resonance and Atomic and Molecular Physics, Wuhan \\ Institute of Physics and Mathematics, Innovation Academy for Precision Measurement Science \\ and Technology, Chinese Academy of Sciences, Wuhan 430071, China \\ ${ }^{2}$ Aix Marseille University, CNRS, ICR, Marseille, France

\section{* Corresponding authors}

Xinli Song - Email: xinli.song@wipm.ac.cn

Josene M. Toldo - Email: josene-maria.TOLDO@univ-amu.fr

Mario Barbatti - Email: mario.barbatti@univ-amu.fr; Website: www.barbatti.org 


\begin{abstract}
A recent experimental study with time-resolved velocity map imaging demonstrated that the total kinetic energy release spectra obtained from photodissociation of ortho and meta fluorophenols have distinct features after excitation into the origin of the $S_{1}$ state. A peak at 6000 $\mathrm{cm}^{-1}$ was observed for both molecules, while another at $13000 \mathrm{~cm}^{-1}$ dominates the spectrum of ortho-fluorophenol. The peak at $6000 \mathrm{~cm}^{-1}$ was assigned to $\mathrm{H}$ tunneling. Nevertheless, the 13000 $\mathrm{cm}^{-1}$ feature remains unassigned. In this work, we performed a theoretical analysis, investigating two hypotheses for explaining the $13000 \mathrm{~cm}^{-1}$ signal. The first hypothesis is that it is due to onephoton absorption followed by ionization through resonant multiphoton dissociation. The second hypothesis is that the signal is due to two-photon absorption into a superexcited state, which dissociates yielding an $\mathrm{H}$ atom. We discuss the pros and cons of each hypothesis, laying the groundwork for future experiments.
\end{abstract}

\title{
TOC Figure
}

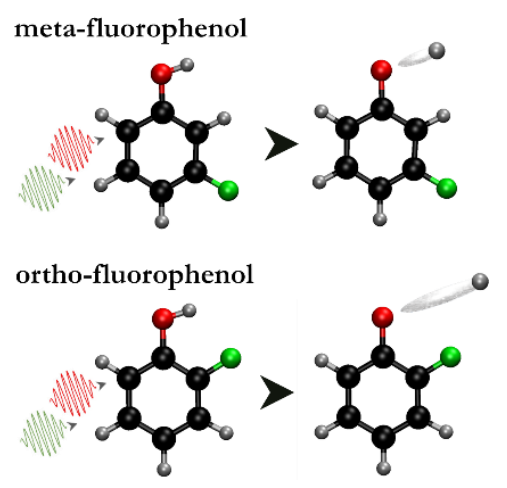

Keywords: Theoretical chemistry; Superexcited states; Dissociative ionization; Time-resolved spectroscopy; Photodissociation; Total kinetic energy release 
Tunneling is one of the most intriguing quantum mechanical phenomena, ${ }^{1}$ and the observation of hydrogen dissociation in the photolysis dynamics of phenol and its derivatives has been a crucial source of information on molecular tunneling mechanisms..$^{2-14}$ It is well established ${ }^{3,}$ ${ }^{15-17}$ that the photolysis of these systems occurs along a ${ }^{1} \pi \pi^{* / 1} \pi \sigma^{*}$ pathway and follows two regimes. When the ${ }^{1} \pi \pi^{*}$ state of the phenol derivative is excited above the ${ }^{1} \pi \pi^{*} /{ }^{1} \pi \sigma^{*}$ energy barrier, the $\mathrm{O}-\mathrm{H}$ bond fission is ultrafast within a few hundred $\mathrm{fs},{ }^{18}$ classically driven by the adiabatic $S_{1}$-state energy. Nevertheless, when the ${ }^{1} \pi \pi^{*}$ state is excited below the energy barrier, the $\mathrm{O}-\mathrm{H}$ fission is mediated by tunneling, occurring in the ns time scale. ${ }^{5}$

Recently, some of us have reported the photolysis dynamics of meta-fluorophenol ( $\mathrm{m}$ $\mathrm{FPhOH})$ and ortho-fluorophenol $(o-\mathrm{FPhOH})$ using time-resolved velocity map imaging (TR-VMI) and time-resolved ion-yield (TR-IY) techniques. ${ }^{2}$ As expected, after excitation of $m$-FPhOH into the origin of the ${ }^{1} \pi \pi^{*}$ state, TR-IY showed that $m$-FPhOH ${ }^{+}$decayed within 2 ns, while TR-VMI showed a complementary birth of $\mathrm{H}^{+}$with the total kinetic energy release (TKER) of $\sim 6000 \mathrm{~cm}^{-1}$, as illustrated in Figure 1-bottom. Such results - which have also been reported by Ashfold group before ${ }^{10}$ - can be directly interpreted as a consequence of tunneling through the ${ }^{1} \pi \pi^{*} /{ }^{1} \pi \sigma^{*}$ energy barrier happening at $2 \mathrm{~ns}$.

In the case of $o-\mathrm{FPhOH}$, TR-IY showed that $o-\mathrm{FPhOH}^{+}$decayed within $3 \mathrm{~ns}$. Other groups have also reported times of this order for the ortho species, in a range from 2.3 to 4.6 ns. ${ }^{19-20}$ Nevertheless, the TR-VMI results in Ref. ${ }^{2}$ revealed $\mathrm{H}^{+}$production occurring before this time range, with a broad TKER signal centered at $\sim 13000 \mathrm{~cm}^{-1}$ (Figure 1-top). Due to the limited experimental information and the lack of detailed theoretical analysis, the underlying mechanism for the 13000 $\mathrm{cm}^{-1}$ TKER signal has not been explained in Ref. ${ }^{2}$ and remains unclear as far as we know. 

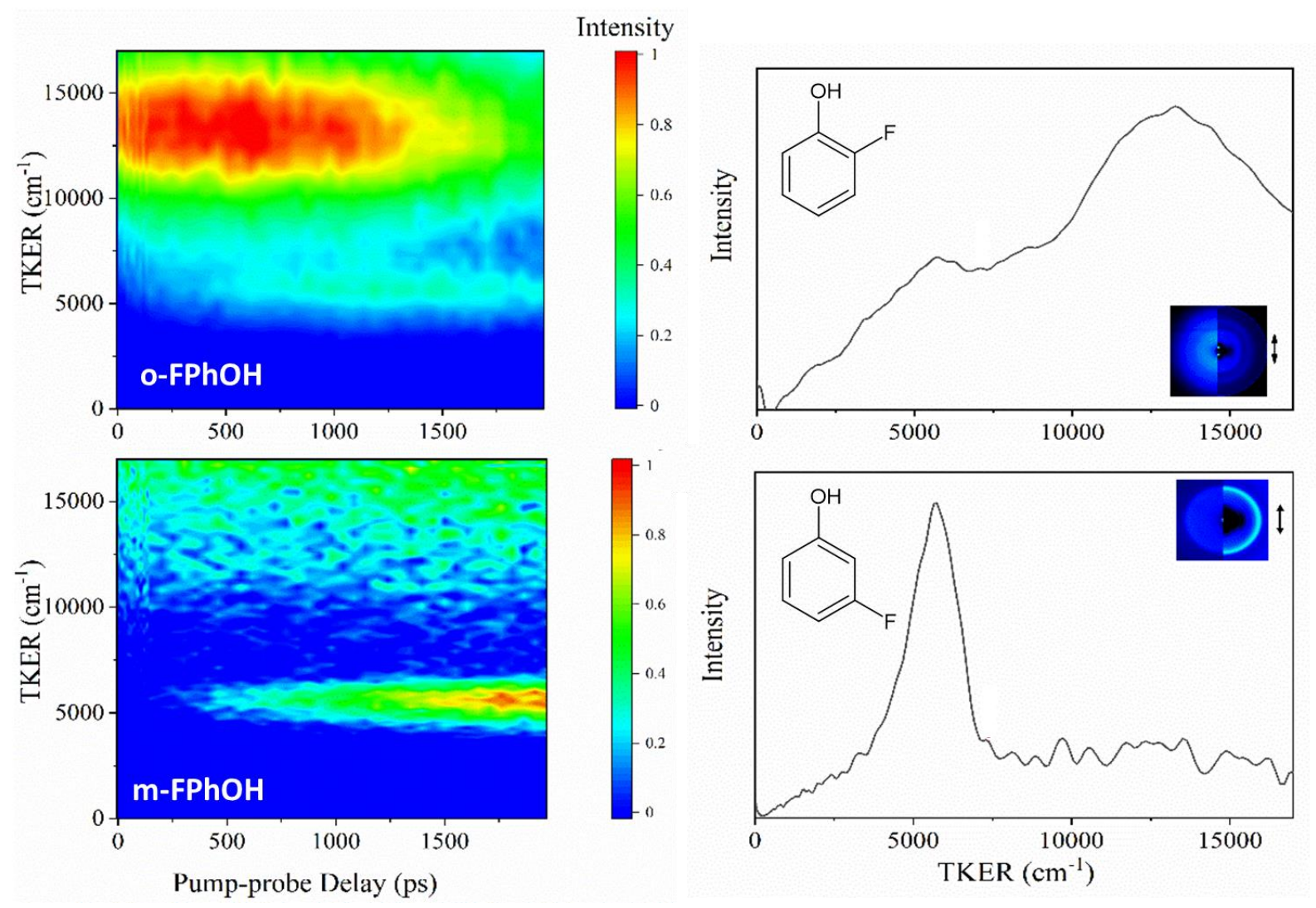

Figure 1. Time-resolved TKER spectrum of $o$-FPhOH (up) and $m$-FPhOH (down) derived from $\mathrm{H}^{+}$velocity map images from Ref. ${ }^{2}$, following excitation of $o-\mathrm{FPhOH}$ at $271 \mathrm{~nm}$ and $m$ $\mathrm{FPhOH}$ at $273 \mathrm{~nm}$, and probing both with $243.1 \mathrm{~nm}$. The inset in the right panels is a representative $\mathrm{H}^{+}$velocity map image recorded after excitation at the origin of ${ }^{1} \pi \pi^{*}$ at a pump-probe delay of 2.0 ns.

Measuring the kinetic energy of the $\mathrm{H}^{+}$fragments, one can discern which $\mathrm{H}$-atoms are created through ${ }^{1} \pi \sigma^{*}$-mediated dissociation, by correlating them to particular features in the TKER spectrum. The $\mathrm{H}^{+}$transient signal can be constructed by integrating the signal associated with the ${ }^{1} \pi \sigma^{*}$ features in the derived TKER spectra, at a series of delay times, which reflects the dynamics of the ${ }^{1} \pi \sigma^{*}$-mediated X-H bond cleavage (X usually $\mathrm{O}$ or $\mathrm{N}$ ). ${ }^{3}$ For excitation above the ${ }^{1} \pi \pi^{*} /{ }^{1} \pi \sigma^{*}$ barrier, the direct dissociation typically has a distribution of recoiling $\mathrm{H}^{+}$around $12000 \mathrm{~cm}^{-1}$. 10-11, ${ }^{21-22}$ For instance, para-fluorophenol shows such a signal at excitation with wavelengths shorter than $238 \mathrm{~nm} \cdot{ }^{23}$ On the other hand, for excitation below the barrier, the tunneling regime typically 
yields a Gaussian-like feature around $5500-6500 \mathrm{~cm}^{-1}$. Nevertheless, a fast- $\mathrm{H}^{+}$signal centered at $13000 \mathrm{~cm}^{-1}$ occurring after excitation into the band origin, as observed for $o-\mathrm{FPhOH}$, cannot be assigned to either regime.

In this letter, we tackled the assignment of the $13000 \mathrm{~cm}^{-1}$ signal from the theoretical point of view. After gathering information about the potential energy surfaces of $o$ - and $m-\mathrm{FPhOH}$ and examining several combinations of absorption of multiple pump and probe photons, we devised two competing hypotheses to explain it:

Hypothesis I: dissociative ionization. After one-photon absorption at the origin of the ${ }^{1} \pi \pi^{*}$ state, resonant two-photon ionization of $o-\mathrm{FPhOH}$, using its first excited cation state $\left(\mathrm{D}_{1}\right)$ as intermediate, leads to fast $\mathrm{H}^{+}$fragments.

Hypothesis II: superexcited states. Two-photon absorption excites $o$-FPhOH into a highlying excited state, which dissociates into a radical and $\mathrm{H}$ atom, and the probe pulses later create fast $\mathrm{H}^{+}$fragments.

Both dissociative ionization and superexcited states have been reported before, demonstrating that ${ }^{1} \pi \sigma^{*}$-mediated $\mathrm{X}-\mathrm{H}$ bond fission is not the only source of $\mathrm{H}^{+}$in substituted phenols and similar systems. Dissociative ionization of the parent molecule has been identified as a source of $\mathrm{H}^{+}$in syringol ${ }^{4}$ (a phenol derivative) and pyrrole. ${ }^{24}$ Fragmentation due to multiphoton activation of superexcited states has been reported to yield $\mathrm{H}^{+}$in phenol. ${ }^{25-26}$ Both processes have been given as possible explanations for the source of fast $\mathrm{H}^{+}$in ethyl ferrulate. ${ }^{27}$ Nevertheless, if either of these processes is the source of fast $\mathrm{H}^{+}$in $o-\mathrm{FPhOH}$, we are in the presence of an unusual situation. Both dissociative ionization and superexcited state processes have always been associated with less intense signals. Usually, they are not even explored in detail, only mentioned as a possible source of some minor unintended signals in the experiments. In $o-\mathrm{FPhOH}$, however, the process responsible for the fast $\mathrm{H}^{+}$signal overshadows the tunneling signal.

Here we examine the details of both hypotheses, as well as their pros and cons. Let us, first, remark that our analysis is based on theoretical calculations with multistate complete active space perturbation theory to second-order (MS-CASPT2) optimizations for both molecules (see Computational Methods). In Figure 2, we show the $\mathrm{S}_{1}$ and $\mathrm{S}_{0}$ potential energy profiles for O-H dissociation of $o$ - and $m$-FPhOH computed with MS-CASPT2. These potential energy profiles 
start from the equilibrium geometry of $S_{1}$ in the ${ }^{1} \pi \pi^{*}$ region, unlike most of the previous studies about halogen-substituted phenols, which draw the profile from the minimum of $\mathrm{S}_{0}$. Our choice follows the reasoning that, in the tunneling regime, the molecule has enough time to relax at this minimum. We have built the dissociation profiles through a rigid elongation of $\mathrm{O}-\mathrm{H}$ distance (which implies the tunneling itself is supposed to be faster than vibrational relaxation).

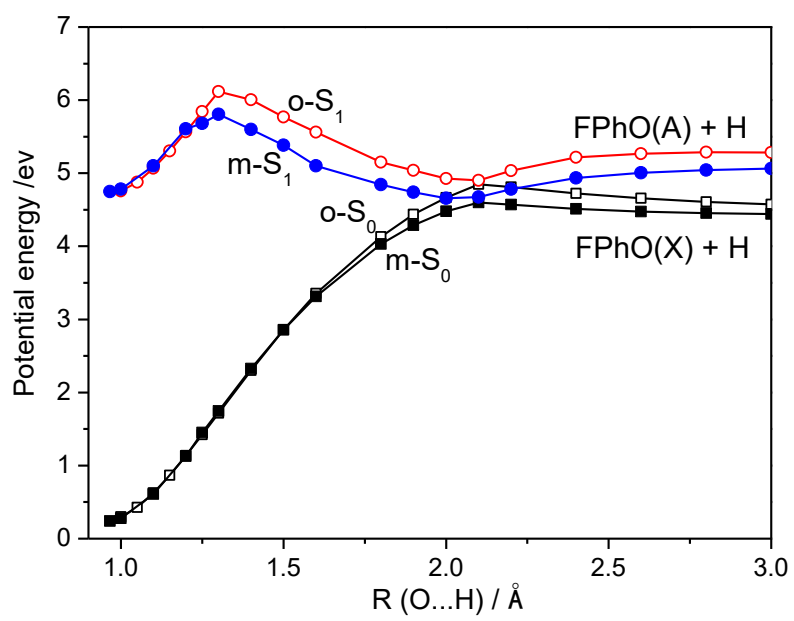

Figure 2. MS-CASPT2 energy profiles along the rigid $\mathrm{O}-\mathrm{H}$ bond fission starting from the $\mathrm{S}_{1}$ minimum. Open symbols: $o$-FPhOH; filled symbols: $m-\mathrm{FPhOH}$.

We have also scanned the potential energy profile along the $\mathrm{CCOH}$ dihedral angle (see Supporting Information SI-1). The stability analysis confirmed that the cis (syn) isomer of $o$ $\mathrm{FPhOH}$ dominates the population due to the interaction between the hydroxyl group and fluorine. ${ }^{19}$ For $m-\mathrm{FPhOH}$, cis is still dominant, but we may expect an appreciable amount of trans (anti) isomer due to the lack of hydrogen bond. Regardless, the photochemistry of the meta species should be independent of the isomer. Thus, we only consider the cis isomer in the computational analysis of both meta and ortho molecules.

The potential energies of the lowest neutral and cation states computed at different geometries are summarized in Table 1. All geometries were optimized with MS-CASPT2 with an active space, including 10 electrons in 9 orbitals and state-averaged over three states (Cartesian coordinates are given in SI-2). From a technical standpoint, we notice that it is crucial to consider 
dynamical electron correlation in the optimization. Without it, it is not possible to describe the outof-plane distortion of the minimum of the ${ }^{1} \pi \pi^{*}$ state of both molecules. With, CASSCF optimization, for instance, the minima are planar. Note, however, that the out-of-plane distortion found with MS-CASPT2 optimization is not as strong as that predicted by TDDFT optimization. ${ }^{28}$

Table 1 Neutral and cation state energies (in $\mathrm{eV}$ ) of the ortho and meta species for the $\mathrm{S}_{0}$, $\mathrm{S}_{1}$, and dissociated geometries. Computed with MS-CASPT2/6-311G**.

\begin{tabular}{|c|c|c|c|c|c|c|}
\hline \multirow[b]{2}{*}{ state } & \multicolumn{3}{|l|}{ Ortho } & \multicolumn{3}{|l|}{ Meta } \\
\hline & $\mathrm{S}_{0}$ geom. & $\mathrm{S}_{1}$ geom. & Diss. geom. & $\mathrm{S}_{0}$ geom. & $\mathrm{S}_{1}$ geom. & Diss. geom. \\
\hline $\mathrm{S}_{0}$ & 0.00 & 0.27 & 4.01 & 0.00 & 0.24 & 3.99 \\
\hline $\mathrm{S}_{1}\left({ }^{1} \pi \pi^{*}\right)$ & 4.91 & 4.73 & 5.20 & 4.96 & 4.75 & 5.03 \\
\hline $\mathrm{S}_{2}$ & 6.65 & 7.48 & - & 6.52 & 7.29 & - \\
\hline \multirow[t]{2}{*}{$\mathrm{D}_{0}$} & 8.76 & 8.87 & 13.26 & 8.82 & 8.86 & 13.22 \\
\hline & & & {$[12.36]^{a}$} & & & {$[12.66]^{a}$} \\
\hline $\mathrm{D}_{1}$ & 9.49 & 9.66 & - & 9.52 & 9.66 & - \\
\hline $\mathrm{D}_{2}$ & 11.82 & 11.82 & _- & 11.78 & 11.74 & _ \\
\hline
\end{tabular}

${ }^{a}$ With ground-state optimized dissociated geometry.

We begin our discussion with $m$-FPhOH, whose TKER spectrum is well understood. The process is illustrated in Figure 3. Experimentally, the 0-0 origin of the ${ }^{1} \pi \pi^{*}$ state is at $273.0 \mathrm{~nm}$ for the cis and $272.0 \mathrm{~nm}$ for the trans isomers. ${ }^{10,29}$ Following the excitation of this band with 273 nm pump (Figure 3-A), the probe wavelength, fixed at $243.1 \mathrm{~nm}$, ionizes $\mathrm{H}$ atoms through a $2+1$ resonance-enhanced multiphoton ionization (REMPI) process (Figure 3-C). ${ }^{30} \mathrm{H}^{+}$is then detected using VMI, yielding the peak at $6000 \mathrm{~cm}^{-1}$ in Figure 1-bottom. From the computational side, we can estimate that the TKER maximum is at $h v_{\text {pump }}-\Delta_{0}(\mathrm{O}-\mathrm{H})$, where $\Delta_{0}(\mathrm{O}-\mathrm{H})$ is the adiabatic O-H dissociation energy. With MS-CASPT2 (Table 1), $\Delta_{0}$ is $3.99 \mathrm{eV}$, corresponding to a $\mathrm{TKER}_{\max }$ at $4449 \mathrm{~cm}^{-1}$, about $1500 \mathrm{~cm}^{-1}$ below the center of the experimental value in Figure 1bottom. Note, however, that we are dealing with the uncertainty of the computational methods. If 
$\Delta_{0}$ is estimated with density functional theory, we obtain $3.64 \mathrm{eV}$ (PBE0/aug-cc-pVTZ), and the TKER $_{\max }$ is predicted to be at $7276 \mathrm{~cm}^{-1}, 1200 \mathrm{~cm}^{-1}$ above the experimental result. Thus, we take such values around 5000 to $7000 \mathrm{~cm}^{-1}$ as a fair agreement with the experiment.

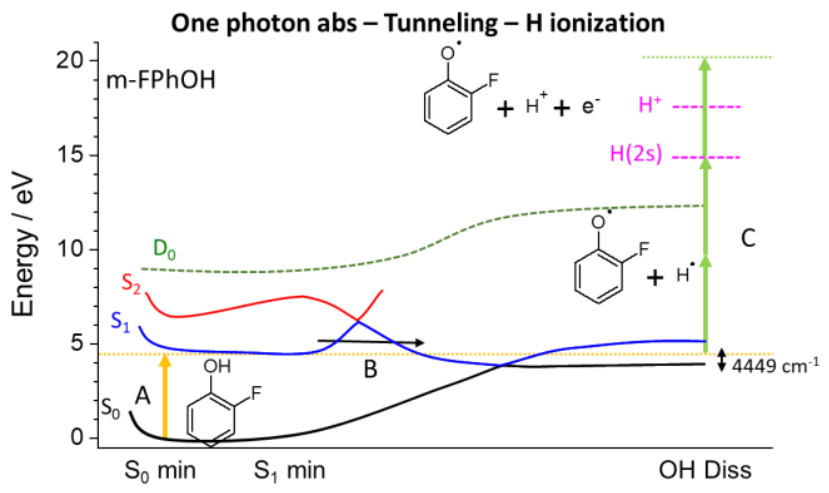

Figure 3. Mechanism of $\mathrm{H}^{+}$formation through tunneling in the $6000 \mathrm{~cm}^{-1}$ TKER signal in $m$ $\mathrm{FPhOH}$. At the stationary points, the states have MS-CASPT2 energies. Schematic potential energy curves connect them. A similar potential energy profile for $o-\mathrm{FPhOH}$ is shown in SI-3.

In the case of $o-\mathrm{FPhOH}$, the $\mathrm{H}^{+}$peak shifts to earlier times, yielding the broad TKER feature at $13000 \mathrm{~cm}^{-1}$ (Figure 1-top). Our first hypothesis (hypothesis I) to explain this peak is illustrated in Figure 4-top. In the case of $o-\mathrm{FPhOH}$, the experimental 0-0 origin of the ${ }^{1} \pi \pi^{*}$ state is at $271.7 \mathrm{~nm}$ for the cis and $271.0 \mathrm{~nm}$ for the trans isomers. ${ }^{19,31}$ Due to the difference of stability, only cis is relevant. Initially, $o-\mathrm{FPhOH}$ is excited by one pump photon $(271.0 \mathrm{~nm})$ into the origin of ${ }^{1} \pi \pi^{*}$ (Figure 4-top-A). Then, two probe photons $(2 \times 243.1 \mathrm{~nm})$ ionize $o$-FPhOH using $\mathrm{D}_{1}$ as an intermediary state for the resonance (B). After ionization, the $o-\mathrm{PhOH}^{+}$cation dissociates into the ground-state radical and $\mathrm{H}^{+}(\mathrm{C})$.

The crucial point of hypothesis $\mathrm{I}$ is the resonant two-photon ionization at $2 \times 243.1 \mathrm{~nm}$ $(2 \times 5.1 \mathrm{eV})$. According to MS-CASPT2, the first excited state of the cation, $\mathrm{D}_{1}$, is $4.93 \mathrm{eV}$ above the minimum of the ${ }^{1} \pi \pi^{*}$ state (Table 1$)$. If we consider the error bar in the calculations $(0.2 \mathrm{eV})$, the $\mathrm{D}_{1}$ state could be an intermediary state for the $(1+1)$ resonance at $243.1 \mathrm{~nm}(5.1 \mathrm{eV})$. If this is the case, the full dissociation process would require only three photons, 1 pump +2 probe, and it could also happen in short times, from the minimum of the ${ }^{1} \pi \pi^{*}$ state. The total kinetic energy 
release is calculated to be $19481 \mathrm{~cm}^{-1}$ (the 3 photons minus the relaxed energy of the dissociated $\mathrm{D}_{0}, 12.36 \mathrm{eV}$ ), which means that about $6000 \mathrm{~cm}^{-1}$ should be deposited in the departing electron.

We recall that a valid hypothesis must explain not only the origin of the fast $\mathrm{H}^{+}$in the TRVMI, but also the 3-ns decay of $o-\mathrm{FPhOH}^{+}$in the TR-IY. According to the hypothesis I, $o$-FPhOH is still excited to the $0-0{ }^{1} \pi \pi^{*}$ origin, and for this reason, it may undergo $\mathrm{H}$ tunneling, which would lead to the $o-\mathrm{FPhOH}^{+}$decay in the TR-IY within $3 \mathrm{~ns}$. As a result, we may expect that after tunneling, the probe should yield slow $\mathrm{H}^{+}$atoms. In fact, the TKER at later times (>1900 ns) shows the emergence of a $6000-\mathrm{cm}^{-1}$ peak, as we can see in Figure 6 of Ref. ${ }^{2}$
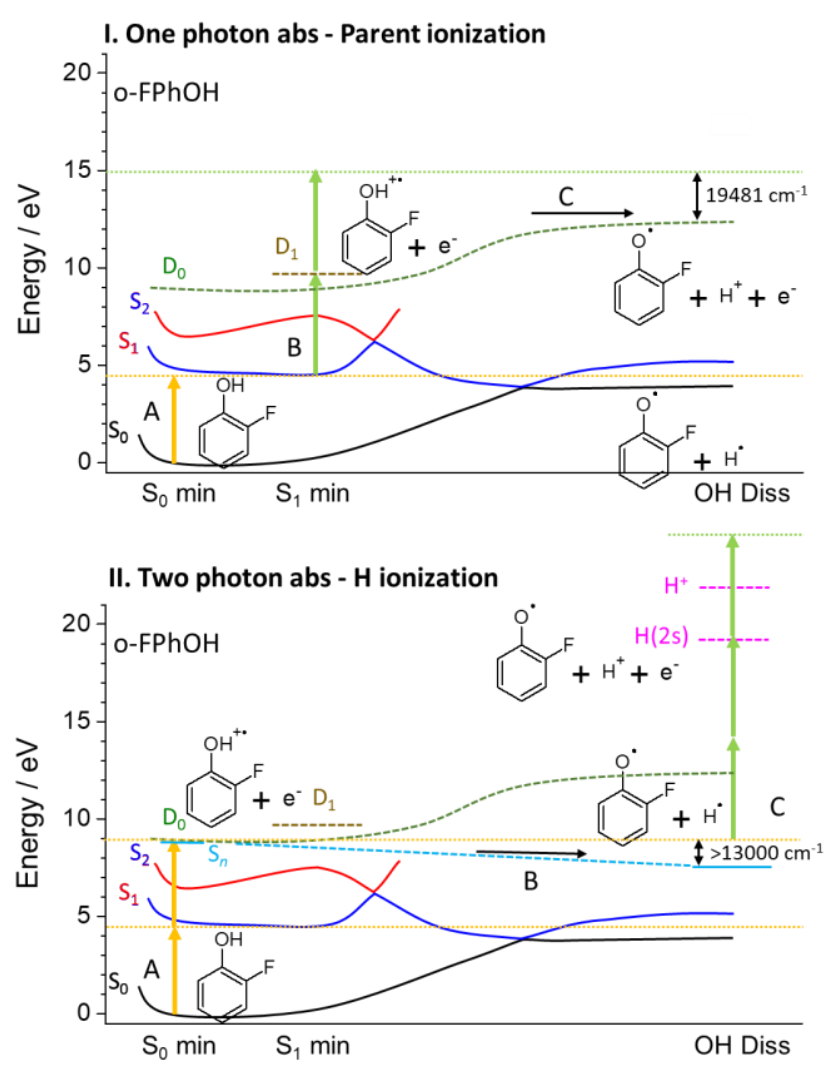

Figure 4. Illustration of the hypothesis to explain the $13000 \mathrm{~cm}^{-1}$ TKER signal in $o-\mathrm{FPhOH}$ based on the MS-CASPT2 values listed in Table 1. Top: Hypothesis I, dissociative ionization. Bottom: Hypothesis II, superexcited state. Similar potential energy profiles for $m$-FPhOH are shown in SI-3. 
Two points go against hypothesis I (but do not invalidate it). First, our calculations showed that the $\mathrm{D}_{1}$ minimum is more stable than the dissociated $\mathrm{D}_{0}$ state (Figure 4-top). Thus, the $\mathrm{H}^{+}$ dissociation is thermodynamically unfavorable, but not impossible. We could also not find a compelling reason for why $m$-FPhOH would also not dissociate through this mechanism. In the case of this molecule, $\mathrm{D}_{1}$ is $4.91 \mathrm{eV}$ above the ${ }^{1} \pi \pi^{*}$ minimum, making the resonance with the 5.1 probe photon slightly less favorable than it is for $o-\mathrm{PhOH}$.

Hypothesis II is illustrated in Figure 4-bottom. Initially, $o-\mathrm{FPhOH}$ is excited by two pump photons $(2 \times 271 \mathrm{~nm})$ into a superexcited state (A). After that, $o$-FPhOH dissociates into an excited radical and $\mathrm{H}(\mathrm{B})$. Finally, $\mathrm{H}^{+}$is generated via $(2+1)$ REMPI $(\mathrm{C})$.

The pros of hypothesis II are that the $\mathrm{H}$ dissociation is thermodynamically favorable, and it could happen in short times. Moreover, the two-photon absorption should access a superexcited state at $9.1 \mathrm{eV}$. The search for states in this energy range revealed that the $\mathrm{O} 3 \mathrm{~s}$ Rydberg state lies at $9.11 \mathrm{eV}$ (SI-4). In hypothesis II, the 3-ns signal in the TR-IY would emerge indirectly, through the fraction of the population still being excited by a single photon into the origin of the $\pi \pi^{*}$ state.

Nevertheless, although feasible, hypothesis II also suffers from a few disadvantages. First, it is based on two sequential multiphoton processes, requiring 5 photons in total. Such a low probability process may be hard (but not impossible) to reconcile with the intense $13000 \mathrm{~cm}^{-1}$ signal. Finally, as in hypothesis I, there is no significant difference between $o$ - and $m$-FPhOH that could explain why the superexcited state process would not occur in the latter. According to the MS-CAPT2 estimate, the O 3s Rydberg state of $m$-FPhOH lies at $8.91 \mathrm{eV}$ (SI-4), slightly offresonance with the pump laser.

At this point, both hypotheses have similar credence, and we are not in the condition of ruling any of them out without further experimental data. One could test hypothesis II by checking whether the intensity of the $13000 \mathrm{~cm}^{-1}$ signal drops with the square of the intensity, as expected for two-photon absorption. ${ }^{32}$ In the case of hypothesis I, as we discussed, the one-photonabsorption process should produce electrons with about $6000 \mathrm{~cm}^{-1}$ kinetic energy. Therefore, some combination of photoelectron angular distributions information from TR-PES with TR-VMI and TR-YI results could provide a way to verify this hypothesis. Finally, one could test both hypotheses by detuning the pump and probe. ${ }^{27}$ If the fast $\mathrm{H}^{+}$signal in $o-\mathrm{FPhOH}$ disappears by changing the 
pump wavelength, it would mean that the resonance with the $\mathrm{O} 3 \mathrm{~s}$ state was lost, favoring hypothesis II. If, however, the fast $\mathrm{H}^{+}$signal disappears by changing the probe wavelength, it would mean that the resonance with the $\mathrm{D}_{1}$ state was lost, favoring hypothesis I.

To summarize, previous experimental work ${ }^{2}$ has shown that $o-\mathrm{FPhOH}$ and $m$-FPhOH have similar TR-IY but completely distinct TKER spectra after excitation into the origin of the ${ }^{1} \pi \pi^{*}$ state, with both decaying after a few ns, but only the ortho species yielding high kinetic energy $\mathrm{H}^{+}$. Based on an MS-CASPT2 analysis of the potential energy profiles of these molecules, we proposed two hypotheses to rationalize the origin of this high kinetic-energy signal. The first hypothesis is based on a one-photon absorption followed by resonant two-photon dissociative ionization of the parent molecule. The second hypothesis is based on two-photon absorption into a superexcited state, followed by dissociation and resonant ionization of $\mathrm{H}$ atoms. Given the pros and cons of each one, we could not rule any of them out. We have also determined probable reasons for the difference between $o$ - and $m$-FPhOH TKER, but not beyond the error bar of the theoretical methodology. Finally, we have proposed tests that could be used to verify each hypothesis. Thus, the present work offers some tantalizing hints for future experimental work.

\section{Computational Methods}

The $\mathrm{S}_{0}, \mathrm{~S}_{1}\left(\pi \pi^{*}\right)$, and $\mathrm{D}_{0}$ structures were optimized at three different levels, namely, density functional theory with PBE0 functional ${ }^{33}$ and aug-cc-pVTZ basis set, ${ }^{34}$ state-averaged complete active space self-consistent (SA-CASSCF) with the $6-311 \mathrm{G}^{* *}$ basis set, ${ }^{35}$ and multistate complete active space perturbation theory to second-order (MS-CASPT2) also with the $6-311 \mathrm{G}^{* *}$ basis set. The dissociated geometries were optimized at the MS-CASPT2/6-311G** level, too.

For SA-CASSCF and MS-CASPT2, the active space was composed of 10 electrons in 9 orbitals, being the $3 \pi$ and $3 \pi^{*}$ of the ring, one $\sigma / \sigma^{*}$ orbital pair in the $\mathrm{O}-\mathrm{H}$ bond, and the oxygen $\mathrm{p}$ orbital parallel to the $\pi$ system (see SI-5). In the MS-CASPT2 calculations, the IPEA parameter was set to $0.25 \mathrm{au}$, and an imaginary level shift of 0.1 a.u was applied to deal with intruder states. The SA-CASSCF was done using three states in the state-averaging procedure.

Separate calculations were done to describe the Rydberg states. The active space was increased to 10 electrons in 10 orbitals, by including the $3 \mathrm{~s}$ orbital centered at the oxygen atom 
(SI-5). In this case, we have used the ANO-RCC-VDZP basis set from OpenMolcas, ${ }^{36}$ augmented with a set of diffuse basis (8s, 8p, 8d). This diffuse set was generated using the OpenMolcas module GENANO and placed at the center of charge of the molecule. Single-point calculations using up to 15 states were done using the MS-CASPT2/6-311G** optimized geometries for the ground state.

All electronic structure calculations were carried out using Gaussian $09^{37}$ and OpenMolcas ${ }^{36}$ program packages.

\section{Associated content}

\section{Supporting Information}

Potential energy profiles; Cartesian coordinates and geometries; illustration of dissociation mechanism; high-energy spectrum; molecular orbitals in the active spaces.

\section{Author Information}

\section{Authors}

Xinli Song: orcid.org/0000-0003-1828-6097

Josene M. Toldo: orcid.org/0000-0002-8969-6635

Carlos E. V. de Moura: orcid.org/0000-0003-1259-3336

Ying Tang: orcid.org/0000-0002-5464-5790

Bing Zhang: orcid.org/0000-0001-8724-1752

Mario Barbatti: orcid.org/0000-0001-9336-6607

\section{Note}

The authors declare no competing financial interest. 


\section{Acknowledgments}

This work was financially supported by the National Key Research and Development Program of China (No.2019YFA0307700) and the National Natural Science Foundation of China (Grant Nos. 21503270, and 11574351, 11774385, 21773299, 11974381, 11674355, 21573279, 21873114) and the China Scholarship Council. The authors thank the project Equip@ Meso (ANR10-EQPX-29-01) funded by the French Government "Investissements d'Avenir" program. MB and JMT thank the FetOpen grant BoostCrop (Grant agreement 828753). CEVM and MB additionally thank funding from ANR through the PRC project WSPLIT (ANR-17-CE05-0005$01)$.

\section{References}

(1) Ramos, R.; Spierings, D.; Racicot, I.; Steinberg, A. M. Measurement of the time spent by a tunnelling atom within the barrier region. Nature 2020, 583, 529-532.

(2) Deng, X.; Tang, Y.; Song, X.; Liu, K.; Gu, Z.; Zhang, B. Photolysis dynamics of m- and o-fluorophenol: Substitution effects on tunneling mechanism. Chemosphere 2020, 253, 126747.

(3) Roberts, G. M.; Stavros, V. G. The role of $\pi \sigma^{*}$ states in the photochemistry of heteroaromatic biomolecules and their subunits: insights from gas-phase femtosecond spectroscopy. Chemical Science 2014, 5, 1698-1722.

(4) Young, J. D.; Staniforth, M.; Dean, J. C.; Roberts, G. M.; Mazzoni, F.; Karsili, T. N. V.; Ashfold, M. N. R.; Zwier, T. S.; Stavros, V. G. Towards Understanding Photodegradation Pathways in Lignins: The Role of Intramolecular Hydrogen Bonding in Excited States. J. Phys. Chem. Lett. 2014, 5, 2138-2143.

(5) Roberts, G. M.; Chatterley, A. S.; Young, J. D.; Stavros, V. G. Direct Observation of Hydrogen Tunneling Dynamics in Photoexcited Phenol. J. Phys. Chem. Lett. 2012, 3, 348-352.

(6) Woo, K. C.; Kim, S. K. Multidimensional H Atom Tunneling Dynamics of Phenol: Interplay between Vibrations and Tunneling. J. Phys. Chem. A 2019, 123, 1529-1537.

(7) Marchetti, B.; Karsili, T. N. V.; Cipriani, M.; Hansen, C. S.; Ashfold, M. N. R. The near ultraviolet photodissociation dynamics of 2- and 3-substituted thiophenols: Geometric vs. electronic structure effects. J. Chem. Phys. 2017, 147, 013923. 
(8) Horbury, M. D.; Baker, L. A.; Quan, W.-D.; Young, J. D.; Staniforth, M.; Greenough, S. E.; Stavros, V. G. Bridging the Gap between the Gas Phase and Solution Phase: Solvent Specific Photochemistry in 4-tert-Butylcatechol. J. Phys. Chem. A 2015, 119, 11989-11996.

(9) Han, S.; You, H. S.; Kim, S.-Y.; Kim, S. K. Dynamic Role of the Intramolecular Hydrogen Bonding in Nonadiabatic Chemistry Revealed in the UV Photodissociation Reactions of 2Fluorothiophenol and 2-Chlorothiophenol. J. Phys. Chem. A 2014, 118, 6940-6949.

(10) Karsili, T. N. V.; Wenge, A. M.; Marchetti, B.; Ashfold, M. N. R. Symmetry matters: photodissociation dynamics of symmetrically versus asymmetrically substituted phenols. Phys. Chem. Chem. Phys. 2014, 16, 588-598.

(11) Chatterley, A. S.; Young, J. D.; Townsend, D.; Żurek, J. M.; Paterson, M. J.; Roberts, G. M.; Stavros, V. G. Manipulating dynamics with chemical structure: probing vibrationallyenhanced tunnelling in photoexcited catechol. Phys. Chem. Chem. Phys. 2013, 15, 6879-6892.

(12) Hadden, D. J.; Roberts, G. M.; Karsili, T. N. V.; Ashfold, M. N. R.; Stavros, V. G. Competing ${ }^{1} \pi \sigma^{*}$ mediated dynamics in mequinol: $\mathrm{O}-\mathrm{H}$ versus $\mathrm{O}-\mathrm{CH}_{3}$ photodissociation pathways. Phys. Chem. Chem. Phys. 2012, 14, 13415-13428.

(13) King, G. A.; Oliver, T. A. A.; Dixon, R. N.; Ashfold, M. N. R. Vibrational energy redistribution in catechol during ultraviolet photolysis. Phys. Chem. Chem. Phys. 2012, 14, 33383345.

(14) Pino, G. A.; Oldani, A. N.; Marceca, E.; Fujii, M.; Ishiuchi, S.-I.; Miyazaki, M.; Broquier, M.; Dedonder, C.; Jouvet, C. Excited state hydrogen transfer dynamics in substituted phenols and their complexes with ammonia: $\pi \pi *-\pi \sigma *$ energy gap propensity and ortho-substitution effect. $J$. Chem. Phys. 2010, 133, 124313.

(15) Sobolewski, A. L.; Domcke, W.; Dedonder-Lardeux, C.; Jouvet, C. Excited-State Hydrogen Detachment and Hydrogen Transfer Driven by Repulsive ${ }^{1} \pi \sigma^{*}$ States: A New Paradigm for Nonradiative Decay in Aromatic Biomolecules. Phys. Chem. Chem. Phys. 2002, 4, 1093-1100. (16) Xie, C.; Ma, J.; Zhu, X.; Yarkony, D. R.; Xie, D.; Guo, H. Nonadiabatic Tunneling in Photodissociation of Phenol. J. Am. Chem. Soc. 2016, 138, 7828-7831.

(17) Xu, X.; Zheng, J.; Yang, K. R.; Truhlar, D. G. Photodissociation Dynamics of Phenol: Multistate Trajectory Simulations including Tunneling. J. Am. Chem. Soc. 2014, 136, 1637816386. 
(18) Iqbal, A.; Pegg, L.-J.; Stavros, V. G. Direct versus Indirect H Atom Elimination from Photoexcited Phenol Molecules. J. Phys. Chem. A 2008, 112, 9531-9534.

(19) Remmers, K.; Meerts, W. L.; Zehnacker-Rentien, A.; Barbu, K. L.; Lahmani, F. Structural information on the S0 and S1 state of o-fluorophenol by hole burning and high resolution ultraviolet spectroscopy. J. Chem. Phys. 2000, 112, 6237-6244.

(20) Harris, S. J.; Karsili, T. N. V.; Murdock, D.; Oliver, T. A. A.; Wenge, A. M.; Zaouris, D. K.; Ashfold, M. N. R.; Harvey, J. N.; Few, J. D.; Gowrie, S., et al. A Multipronged Comparative Study of the Ultraviolet Photochemistry of 2-, 3-, and 4-Chlorophenol in the Gas Phase. J. Phys. Chem. A 2015, 119, 6045-6056.

(21) Nix, M. G. D.; Devine, A. L.; Cronin, B.; Dixon, R. N.; Ashfold, M. N. R. High resolution photofragment translational spectroscopy studies of the near ultraviolet photolysis of phenol. $J$. Chem. Phys. 2006, 125, 133318.

(22) Iqbal, A.; Cheung, M. S. Y.; Nix, M. G. D.; Stavros, V. G. Exploring the Time-Scales of H-Atom Detachment from Photoexcited Phenol-h6 and Phenol-d5: Statistical vs Nonstatistical Decay. J. Phys. Chem. A 2009, 113, 8157-8163.

(23) Devine, A. L.; Nix, M. G. D.; Cronin, B.; Ashfold, M. N. R. Near-UV photolysis of substituted phenols, I: 4-fluoro-, 4-chloro- and 4-bromophenol. Phys. Chem. Chem. Phys. 2007, 9, 3749-3762.

(24) Lippert, H.; Ritze, H. H.; Hertel, I. V.; Radloff, W. Femtosecond time-resolved hydrogenatom elimination from photoexcited pyrrole molecules. Chemphyschem 2004, 5, 1423-1427.

(25) Schick, C. P.; Weber, P. M. Ultrafast Dynamics in the Three-Photon, Double-Resonance Ionization of Phenol via the $\mathrm{S}_{2}$ Electronic State. The Journal of Physical Chemistry A 2001, 105 , 3735-3740.

(26) Dixon, R. N.; Oliver, T. A. A.; Ashfold, M. N. R. Tunnelling under a conical intersection: Application to the product vibrational state distributions in the UV photodissociation of phenols. The Journal of Chemical Physics 2011, 134, 194303.

(27) Rodrigues, N. D. N.; Staniforth, M.; Young, J. D.; Peperstraete, Y.; Cole-Filipiak, N. C.; Gord, J. R.; Walsh, P. S.; Hewett, D. M.; Zwier, T. S.; Stavros, V. G. Towards elucidating the photochemistry of the sunscreen filter ethyl ferulate using time-resolved gas-phase spectroscopy. Faraday Discuss. 2016, 194, 709-729. 
(28) Ling, F.; Li, S.; Song, X.; Wang, Y.; Long, J.; Zhang, B. Femtosecond time-resolved observation of butterfly vibration in electronically excited o-fluorophenol. Scientific Reports 2017, 7, 15362.

(29) Yosida, K.; Suzuki, K.; Ishiuchi, S.-i.; Sakai, M.; Fujii, M.; Dessent, C. E. H.; MüllerDethlefs, K. The PFI-ZEKE photoelectron spectrum of $\mathrm{m}$-fluorophenol and its aqueous complexes: Comparing intermolecular vibrations in rotational isomers. Phys. Chem. Chem. Phys. 2002, 4, 2534-2538.

(30) Bjorklund, G. C.; Ausschnitt, C. P.; Freeman, R. R.; Storz, R. H. Detection of atomic hydrogen and deuterium by resonant three-photon ionization. Appl. Phys. Lett. 1978, 33, 54-56.

(31) Fujimaki, E.; Fujii, A.; Ebata, T.; Mikami, N. Autoionization-detected infrared spectroscopy of intramolecular hydrogen bonds in aromatic cations. I. Principle and application to fluorophenol and methoxyphenol. J. Chem. Phys. 1999, 110, 4238-4247.

(32) Biczysko, M.; Bloino, J.; Santoro, F.; Barone, V., Time-Independent Approaches to Simulate Electronic Spectra Lineshapes: From Small Molecules to Macrosystems. In Computational Strategies for Spectroscopy, John Wiley \& Sons, Inc.: 2011; pp 361-443.

(33) Adamo, C.; Barone, V. Toward reliable density functional methods without adjustable parameters: The PBE0 model. J. Chem. Phys. 1999, 110, 6158-6170.

(34) Dunning Jr., T. H. Gaussian Basis Sets for Use in Correlated Molecular Calculations. I. The Atoms Boron Through Neon and Hydrogen. J. Chem. Phys. 1989, 90, 1007-1023.

(35) Ditchfie.R; Hehre, W. J.; Pople, J. A. Self-Consistent Molecular-Orbital Methods .9. Extended Gaussian-Type Basis for Molecular-Orbital Studies of Organic Molecules. J. Chem. Phys. 1971, 54, 724-\&.

(36) Fdez. Galván, I.; Vacher, M.; Alavi, A.; Angeli, C.; Aquilante, F.; Autschbach, J.; Bao, J. J.; Bokarev, S. I.; Bogdanov, N. A.; Carlson, R. K., et al. OpenMolcas: From Source Code to Insight. J. Chem. Theory Comput. 2019, 15, 5925-5964.

(37) Frisch, M. J.; Trucks, G. W.; Schlegel, H. B.; Scuseria, G. E.; Robb, M. A.; Cheeseman, J. R.; Scalmani, G.; Barone, V.; Mennucci, B.; Petersson, G. A., et al. Gaussian 09, Revision D.01; Gaussian, Inc.: Wallingford, CT, USA, 2013. 